\title{
MULTIPLE SHELL PLANETARY NEBULAE
}

\author{
You-Hua Chu \\ Astronomy Department, University of Illinois, \\ 1011 W. Springfield Avenue, Urbana, IL 61801 \\ U. S. A.
}

\begin{abstract}
It has been shown in several independent investigations that the multiple-shell phenomenon is prevalent in planetary nebulae. Despite the common classification, the multiple shell planetary nebulae are a heterogeneous group of objects, as testified by the wide variety of their morphologies and physical structures. There are two types of double-shell structures that are seen frequently: one has an inner shell expanding supersonically into a faint, subsonically expanding halo, and the other has a bright attached envelope co-expanding with the inner shell. The physical structures and relative elemental abundances in the shells are reviewed, and their possible formation mechanisms are discussed.
\end{abstract}

\section{INTRODUCTION}

Many planetary nebulae (PNe) have been reported to contain "two concentric rings," "faint envelopes," or "extended halos" by Curtis (1918), Duncan (1937), Minkowski and Osterbrock (1960), and Millikan (1974). A catalog of such nebulae, called "multiple shell planetary nebulae" (MSPNe), has been compiled by Kaler (1974). All of Kaler's MSPNe can be described by: 1) the outer structure is a regular ring that encircles at least half the extent of the inner nebula, and 2) there is a break in the surface brightness profile at the interface of the inner and outer shells, giving the double-shell appearance. In a recent compilation of MSPNe (Chu, Jacoby, and Arendt 1987), 41 nebulae are included, and the number is rapidly growing with the addition of IC 1297, NGC 6751, and many southern MSPNe reported in this symposium (Aller, Keyes, and Feibelman 1986; Gieseking and Solf 1986; Ruiz, Heathcote, and Weller 1987; Lutz, Lame, and Balick 1987).

A30 and A78 have not been included in the list of MSPNe although they have different abundances and expansion velocities in their inner and outer regions, indicating multiple ejections (Jacoby and Ford 1983; Reay, Atherton, and Taylor 1983a; Jacoby and Chu 1987; Pismis and Moreno 1987; Manchado, Pottasch, and Mampaso 1987a). The exclusion is 105 
somewhat arbitrary, and is intended only to narrow down the scope of MSPN studies, since $A 30$ and $A 78$ are clearly at a very different evolutionary stage from the conventional MSPNe (Iben et al. 1983).

There are several candidates for a new kind of MSPN; they have been reported as PNe with extended halos (at a level of $10^{-3}-10^{-4}$ times the peak surface brightness). For example, faint emission lines are still detected at twice the known radius of NGC 1535 (Bässgen et al. 1986; Weller and Heathcote 1987a) and other nebulae (Bässgen et al. 1987). The most convincing evidence for the existence of faint halos comes from the high-dispersion spectroscopic observations: unresolved lines are detected outside the boundary of the fast expanding main nebula of NGC 6210 and NGC 6309 (Chu and Jacoby 1987). It is premature to include such halos in this review; however, they deserve future consideration.

\section{FREQUENCY OF OCCURRENCE}

It is perhaps fortuitous that ALL PNe with well-known names have double shells, for example, the Dumbbell, the Eskimo, the Helix, the Owl, the Ring, and the Saturn nebulae; nevertheless, it is a good indication that the multiple-shell phenomenon is common in PNe.

In the Catalogue of Galactic Planetary Nebulae (Perek and Kohoutek 1967), about $25 \%$ of the resolved PNe have "outer envelopes." In a recent deep CCD image survey of $44 \mathrm{PNe}$, Jewitt, Danielson, and Kupferman (1986) find that $2 / 3$ of them have extended halos, defined as material beyond the 108 isophotal boundaries. Bässgen et al. (1987) find spectroscopically that 15 out of the 20 PNe they studied have halos. Although some of these outer envelopes and extended halos are the lobes of bipolar nebulae (e.g., NGC 650-651 and NGC 6781) and some consist of only irregular wisps and filaments (e.g., NGC 6309 and NGC 6772), most of them do have shell structures. These surveys imply unanimously that a significant fraction of PNe have multiple shells.

A rigorous derivation of the multiple-shell occurrence rate is given by Chu, Jacoby, and Arendt (1987). They have examined the 126 PNe in the New General Catalogue (NGC) and Index Catalogue (IC), and carefully analyzed the selection biases in this sample. They find that in the 56 nearby $(<3 \mathrm{kpc}$ ) optically thin PNe in NGC/IC, at least 28 have multiple shells. They conclude that more than 508 of PNe go through the visible multiple-shell phase during their lifetime.

It should not be surprising that the multiple-shell phenomenon is so prevalent, since the multiple-shell appearance is indicative of abrupt changes in stellar mass loss during the PN formation, and the mass loss from the central star of a PN does change drastically in both rate and velocity from the progenitor AGB phase to the current PNN phase (cf. Kwok 1987; Cerruti-Sola and Perinitto 1985). 


\section{MORPHOLOGY OF MULTIPLE SHELLS}

In an earlier attempt to classify multiple-shell morphology, Kaler (1974) defined bright double, weak double, giant halo, and triple shell nebulae, according to the relative surface brightness, size, and number of shells in a nebula. As more high-quality images become available, it is clear that some of his weak double shell nebulae (He 1-5, IC 289, and NGC 2610) are just fainter counterparts of the bright doubles (e.g., NGC 1535 and NGC 7354), and the weak double nebula NGC 2438 is morphologically similar to the giant halo nebulae (e.g., NGC 6826 and NGC 6891) except that its outer shell is less than 5 times as large as its inner shell. This problem is remedied in the new classification scheme (Chu, Jacoby, and Arendt 1987) described in the remainder of this section. In the new scheme, the class of weak double nebula is disassembled. Some of the weak doubles are combined with the giant halo nebulae and called Type I; the others are combined with the bright doubles into Type II.

For convenience, only the double-shell morphology is classified below, since nebulae with more than two shells can be decomposed into a small number of double-shell units. The triple shell nebulae may consist of different types of outer and inner double shells. Images of MSPNe can be found in papers by Chu, Jacoby, and Arendt (1987) and Balick (1987a). Some representative examples are given in Figure 1.

Type I is characterized by faint outer shells with noticeable limb-brightening, often called faint halos. The detailed outer shell structure may vary from the smooth and regular cases of NGC 2438 , NGC 6826, NGC 6891, and NGC 7662 to the irregular filamentary cases of He 2-111, NGC 6543, and NGC 6720. About 308 of the known MSPNe have type I double shells.

Type II is characterized by bright outer shells that appear attached to their inner shells. There are different degrees of symmetry in geometry and surface-brightness variation in the type II double-shell morphology. Some nebulae have outer shells with similar thickness in all directions. Among these some have extremely smooth, featureless outer shells (type IIa), for example, IC 3568 and NGC 1535; while the others have significant brightness variation in the outer shells (type IIb). The variation is often amplified in the [N II] line images, for example, M2-2 and NGC 7662. Some nebulae have a marked elongation in the inner shell which seems to be breaching out of the outer shell (type IIC), for example, IC 289, NGC 6058, NGC 6804, NGC 7009, and NGC 7354. Note that there are still differences in surface brightness variation, parallel to the distinction between type IIa and type IIb, among the type IIc nebulae; perhaps further classification is warranted. There are also some peculiar nebulae with such irregular structures in their outer shells that they do not fit in any of the three subclasses above, for example, NGC 1545, NGC 2392, NGC 4361, and NGC 6026. About $70 \%$ of the known MSPNe have type II double shell structures. 

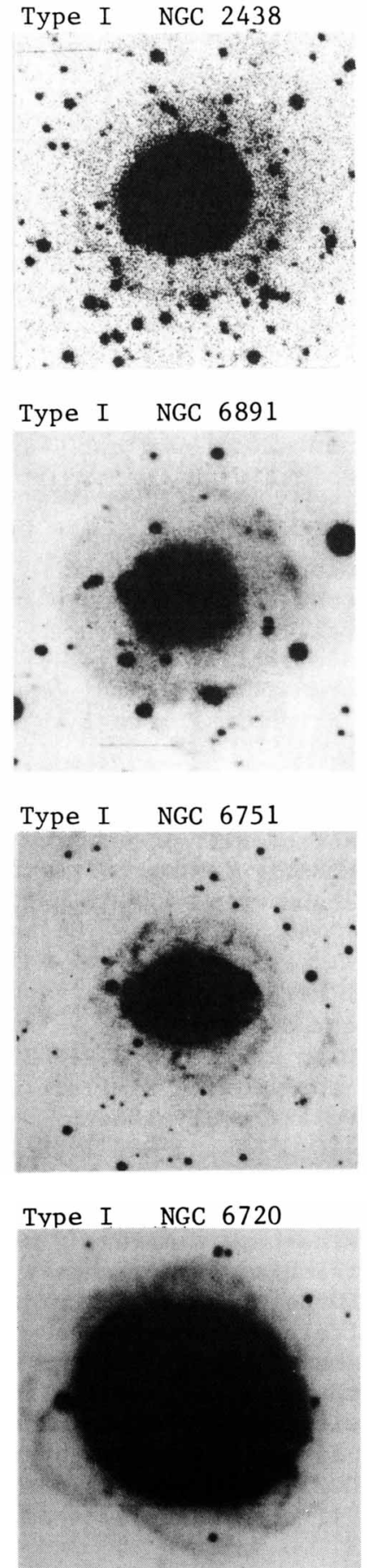

Tvpe IIa NGC 1535

Type IIb NGC 7662

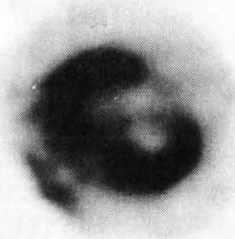

Type IIc IC 289

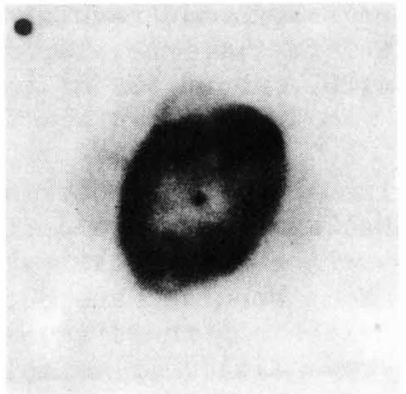

Type IIc NGC 6804

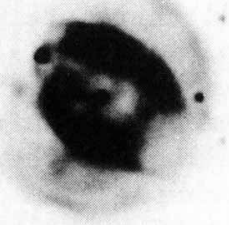

Tvpe IId NGC 4361

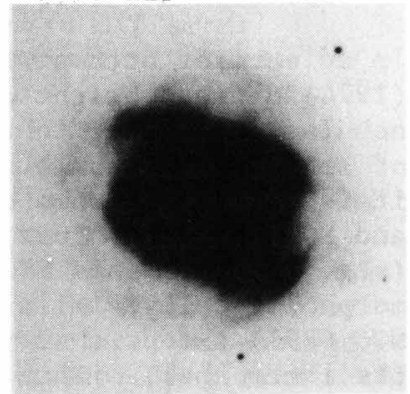

Type P NGC 6369
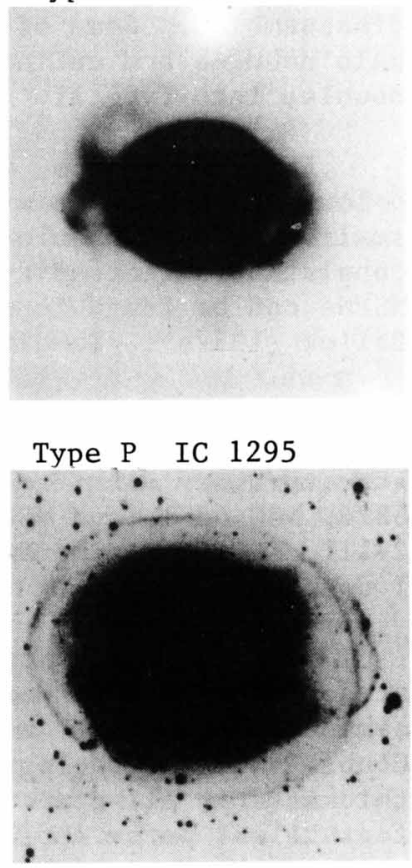

Figure 1.

Examples of different multiple-shell morphologies. 
Finally, there are a small number of nebulae classified "peculiar" for one of the two reasons: 1) The double-shell structure seems to be a hybrid of types I and II. Since only A2 and IC 1295 are in this category, a separate classification is not justified for the time being. 2) The outer shell is so irregular that neither of the two types can describe its structure, for example, NGC 3132, NGC 6369, and the outer shells of NGC 6804. Some of these outer shells may be illuminated interstellar medium, as they seem to be part of the more extended nebulosity in the vicinity, for example, NGC 6857 and NGC 6894.

The two aforementioned morphological classes have very little overlap in properties such as size of the outer shell, relative sizes of the shells, and the relative surface brightness of the shells (Chu, Jacoby, and Arendt 1987). The type I MSPNe usually have outer diameter larger than $0.5 \mathrm{pc}$, while those of the type II MSPNe are smaller than $0.5 \mathrm{pc}$. The type I MSPNe mostly have the ratio of outer to inner radii greater than 2, while for the type II MSPNe it is smaller than 2. The surface brightness of the outer shell is usually less than $5 \times 10^{-3}$ times that of the inner shell for the type I MSPNe, while the type II MSPNe have outer surface brightness about 0.1-0.5 times the inner surface brightness.

There are several triple shell nebulae. Four of them, NGC 2022, NGC 6826, NGC 6891, and NGC 7662, have type II inner double-shells and type I outer double-shells. NGC 3132 has peculiar inner double-shells and type I outer double-shells. NGC 6720 and NGC 6751 contain two type I halos (Moreno and López 1987; Chu and Jacoby 1987). The triple-shell identification may be debatable for NGC 3242, NGC 6804, and NGC 6894; these three nebulae have type II inner double-shells and peculiar outermost structure, of which a circumstellar origin is uncertain (Rosado 1986; Chu, Jacoby, and Arendt 1987).

\section{PHYSICAL STRUCTURES DERIVED FROM INTERNAL MOTIONS}

The most direct way to probe the physical structure of MSPNe is through the study of internal motions. The relative motion of the shells allows us to determine the dynamical evolution and to test critically the PN-formation models. In the high dispersion spectroscopic observations it is essential to resolve the shells spatially, as well as spectrally. Welty's (1983) kinematic study of MSPNe was hampered by his lack of spatial resolution; only integrated Fabry-Perot spectra were obtained, and no definitive conclusion could be reached. Sabbadin (1984, and references therein) obtained long-slit echelle observations for a large number of MSPNe; however, most of the outer shells were undetected. The Eskimo nebula has been extensively studied by Reay, Atherton, and Taylor (1983b), O'Dell and Ball (1985), Gieseking, Becker, and Solf (1985), and Balick, Preston, and Icke (1988); the internal motion of the Eskimo is so complex that no consistent model to explain the kinematic features in both [O III]- and [N II]-lines is available yet. 


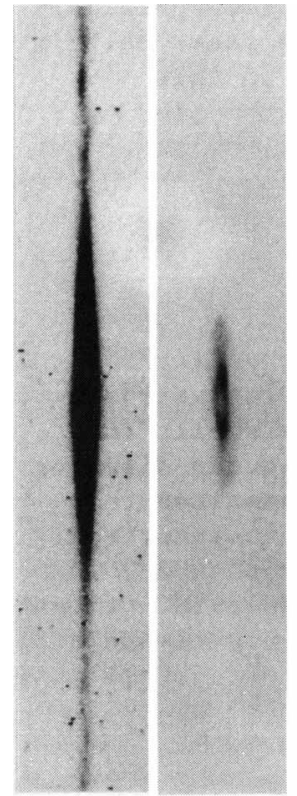

(a)

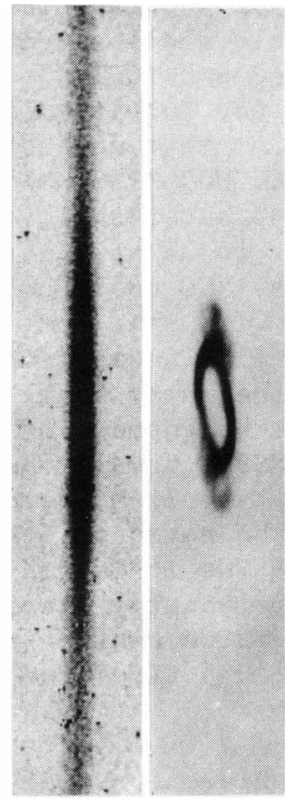

(b)

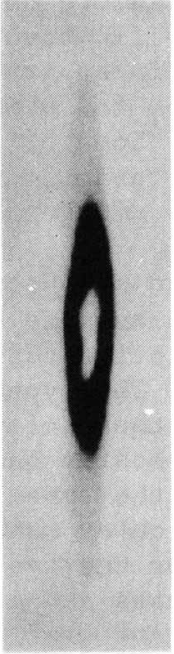

(c)

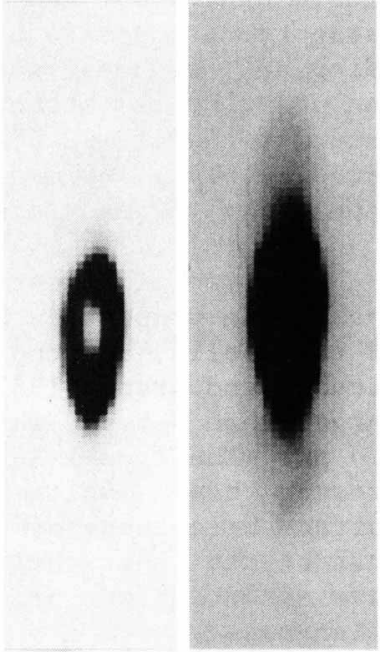

(d)
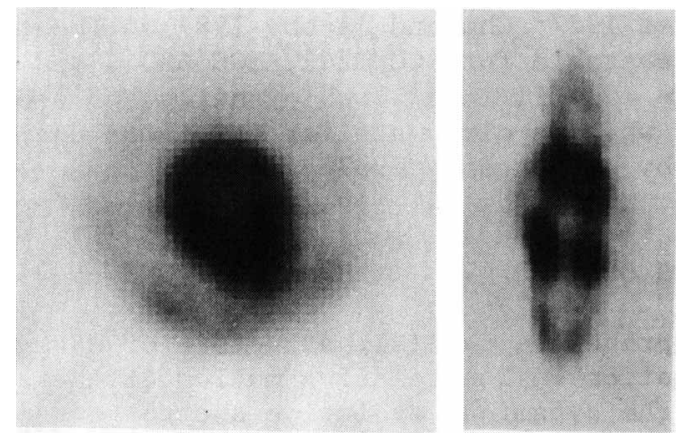

(e)

Figure 2. [O III] echelle line images of MSPNe. a) NGC 6826. The spectrum of the halo is on the left and that of the inner shells along the minor axis is on the right. b) NGC 7662. The spectrum of the halo is on the left, and that of the inner shells along the major axis is on the right. Each panel in a) and b) has $300 \mathrm{~km} / \mathrm{s}$ along the $X$ axis and 130" along the Y-axis. c) NGC 1535. d) IC 3568. Both panels are from the same exposure, windowed differently to show the features at higher or lower brightness levels. e) M2-2. An [0 III] image is on the left, and an echelle line image is on the right. The slit was NS oriented, passing through the center. 
Chu and Jacoby (1988) have obtained long-slit echelle/CCD

observations of 28 MSPNe, with special effort to detect the faint outer shells. The discussion in this section is based mainly on this set of homogeneous data. Some echelle line images are reproduced in Figure 2. The patterns of internal motion, archetypical examples, and their associated morphologies are summarized below:

i) The inner shell expands supersonically into a faint halo which expands subsonically or mildly supersonically.

Example - IC 4593, NGC 6751, NGC 6826, NGC 6891, and NGC 7662. Morphology - type I outer shells.

ii) The outer shell is an attached envelope, co-expanding supersonically with the inner shell. For any nebulae with an ellipsoidal inner shell, the expansion velocity of the outer shell is similar to that of the inner shell along the minor axis, but slower than that along the major axis.

Example - (round) NGC 1535 and NGC 2610.

(ellip.) NGC 2022, NGC 6058, NGC 7009, and NGC 7354. Morphology - (round) type IIa, and (ellip.) type IIc.

iii) The outer shell expands independently from the inner shell. Example - He 2-111*, M2-2, NGC 6720, and the Helix*

Morphology - type I and type IIb.

(*: He 2-111, Webster 1978; the Helix, Walsh and Meaburn 1987)

iv) The outer shell expands faster than the inner shell!

Example - IC 3568, NGC $6826^{*}$, and NGC $6891^{*}$.

Morphology - type IIa and type IIc.

(*: referring to the inner two shells)

v) Peculiar motion.

Example - NGC 2392, NGC 4361, and NGC 6369.

Morphology - type IIp and type $P$.

Three important inferences can be drawn from these results. First, the nebular morphology is not a sufficient condition for the physical structure, hence should not be cited as supporting evidence for particular formation models. For example, IC 3568 and NGC 1535 both have perfectly smooth and symmetric outer shells and have been used to demonstrate the snowplow phase of the two-wind model (Balick 1987a, 1987b). However, as the line images in Figure 2 show, the outer shell of NGC 1535 expands at a velocity similar to that of the inner shel1, while the outer shell of IC 3568 expands faster than its inner shell; neither case can be explained as snowplow action.

Second, there is direct evidence from the echelle line images that most of the outer shells in MSPNe are filled with material, instead of being hollow shells. The strongest evidence comes from the lack of linesplitting in the profiles which are several times the instrumental resolution. A hollow shell would have given rise to a linesplitting of 
twice the expansion velocity. While the lack of linesplit can be due to either large turbulence in the shell (Hippelein, Bässgen, and Grewing 1985) or the presence of material moving tangentially to the line-of-sight, the filled-envelope geometry is inevitable. A supersonic turbulence will disperse the shell and diffuse back to fill the gap between the shells. For a filled-envelope geometry, there is always material moving tangential to the line-of-sight. This situation is seen in both type $I$ and type II outer shells, as illustrated in the halo of NGC 7662 and the outer shell of NGC 1535 (Figure 2). The observed [O III] velocity FWHM in the halo of NGC 7662 is $47 \mathrm{~km} / \mathrm{s}$, while the instrumental profile is only $9.5 \mathrm{~km} / \mathrm{s}$. This result is significantly different from what Hippelein, Bässgen, and Grewing (1985) derived from their large aperture Fabry-Perot observations; their results are probably spurious due to the enormous contamination of the stray light and the lack of spatial resolution.

Third, the internal motion in MSPNs can be used to determine whether they are bipolar nebulae viewed along the poles. Based on the detection of molecular $\mathrm{H}_{2}$ in NGC 6720 (Zuckerman and Gatley 1988) and the morphology of NGC 2438 , it has been suggested that these two nebulae may be bipolar nebulae (Balick 1987a). However, the radial expansion velocity of both halos are much smaller than their inner main nebulae (Chu and Jacoby 1987), contrary to what is expected in bipolar nebulae (Weller and Heathcote 1987b). While never being suspected, the peculiar MSPNe NGC 4361 and NGC 6369 do have kinematic characteristics of bipolar structure. The Helix nebulae, NGC 7293 might be bipolar, too (Walsh and Meaburn 1987).

\section{RELATIVE ABUNDANCES OF THE SHELLS}

Very few spectroscopic investigations of MSPNe have emphasized the detection of both shells. Future observations should be planned to differentiate the shells.

Type I halos are faint, hence only the brightest ones have been observed - NGC 6543 and NGC 6826. These two MSPNe have been studied by Manchado, Pottasch, and Mampaso (1987b) and Middlemass, Clegg, and Walsh (1987). Both conclude that the halo of NGC 6826 has higher electron temperature and lower $\mathrm{He}$ and $\mathrm{N}$ abundances than the inner she11. Nevertheless, they disagree on the abundance variation in NGC 6543 - one group conclude lower abundances in the halo, while the other group conclude uniform abundances throughout the nebula. The origin of the disagreement is in the electron temperature they derive for the inner shell. Given the complicated ionization structure revealed in the images of NGC 6543 (Balick 1987a), it is probably easy to get different temperatures if different regions are sampled.

Four type II double shells have been studied with spatial resolution - the inner two shells of NGC 3242, NGC 7009, and NGC 7662 (Barker 1983, 1985, 1986), and NGC 6826 (Jacoby, Quigley, and Africano 
1987). None of the nebulae, except maybe NGC 7662, show any significant abundance differences between the inner and outer shells. In NGC 7662, there are bright [N II] knots (Balick 1987a) which may have higher nitrogen abundance, although this has not been emphasized by Barker (1986).

\section{FORMATION MECHANISMS OF MULTIPLE SHELL PLANETARY NEBULAE}

Given the various morphological, kinematic, and chemical structures in the shells, the various MSPNe must have been formed differently from one another. Although theoretical calculations of PN evolution have begun to include three stages of mass loss processes (Schmidt-Voigt and Köppen 1987a, b), there is still a long way before the multiple shells can be satisfactorily modeled. Below are tentative suggestions of the formation mechanism for each type of multiple-shell structure.

The faint, subsonically expanding halos undoubtedly correspond to the slow wind from the AGB phase. The double-shell nebulae with a simple inner shell and a halo are good candidates for Kwok's (1983) two-wind formation, since both the morphology and kinematics of the shells are qualitatively consistent with the model predictions. On the other hand, the inner shell could have been created by an enhanced mass loss rate in a "superwind," too.

The "co-expanding envelope"-type outer shells are difficult to explain, since the outer shell seems to be coasting along rather than actively interacting with the inner shell. It could be that the break between the shells was formed by an abrupt change in the mass loss rate of a "superwind." Nevertheless, it remains to be explained how the shells manage to fight diffusion and maintain a steep density gradient at the interface.

The MSPNe that contain two or three independently expanding systems clearly need multiple ejections. In the case of M2-2 (Figure $2)$, the outer and inner shells have different expansion velocities and ellipticities. Such a rapid change (within a few $\times 10^{3} \mathrm{yr}$ ) in the ejection process might be due to a binary core (Iben and Tutukov 1987).

Three known nebulae have outer shells expanding faster than the inner shells. The dynamical timescale (झ radius/expansion velocity) is longer for the inner shell than for the outer shell. It is unlikely that a fast expanding shell sieves through a slower shell; the outer shell must have been preferentially accelerated. The acceleration could be due to simply the diffusion into vacuum (Schmidt-Voigt and Köppen 1987a) or the radiation pressure on the dust (Mathews 1978). At least one of the nebulae, IC 3568, is well-known for its high dust content (Cohen, Harrington, and Hess 1984).

The peculiar MSPNe are hard to understand because of the insufficient data available. Some of them, particularly NGC 4361 and 
NGC 6369, may be bipolar nebulae projected along certain angles.

\section{FUTURE WORK}

Clearly, there is a lot to be done in the future, both observationally and theoretically, in order to understand the MSPNe better. Several investigators have recently obtained a large number of data on morphologies and internal motions. In the future, it is important to model the 3-D structure of individual nebulae and extract quantitative information such as the radial density profile and the velocity law within the shells. More low-dispersion spectroscopic observations are needed to determine the elemental abundances in the shells of MSPNe. Theoretically, models of PN formation with variable wind or binary cores should be pursued further.

\section{REFERENCES}

Aller, L. H., Keyes, C. D., and Feibelman, W. A. 1986, Ap. J., 311,930 .

Balick, B. 1987a, A. J., 94, 671 . . 1987b, this volume.

Balick, B., Preston, H., and Vicke, V. 1988, submitted to A. J.

Barker, T. 1983, Ap. J., 267, 630.

. 1985, Ap. J., 294, 193.

- 1986, Ap. J., 308, 314 .

Bässgen, M. , Bässgen, G., Barnstedt, J., Grewing, M., and Bianchi, L. 1986, Mitt. Astr. Ges., Nr. 67, p. 342.

Bässgen, M., Bässgen, G., Grewing, M., Cerrato, S., and Bianchi, L. 1987 , in this volume.

Cerruti-Sola, M., and Perinotto, M. 1985, Ap. J., 291, 237.

Chu, Y.-H., and Jacoby, G. H. 1987, in this volume. . 1988, in preparation.

Chu, Y.-H., Jacoby, G. H., and Arendt, R. 1987, Ap. J. Supp1., 64, 529.

Cohen, M., Harrington, J. P., and Hess, R. 1984, Ap. J., 283, 687.

Curtis, H. D. 1918, Pub. Lick Obs., 13, 57.

Duncan, J. C. 1987, Ap. J., 86, 496.

Gieseking, F., Becker, I., and Solf, J. 1985, Ap. J. (Letters), 295, L17.

Gieseking, F., and Solf, J. 1986, Astr. Ap., 163, 174.

Hippelein, H. H., Bässgen, M., and Grewing, M. 1985, Astr. Ap., $152,213$.

Iben, I., Jr., Kaler, J. B., Truran, J. W., and Renzini, A. 1983, Ap. J., 264, 605 .

Iben, I., Jr., and Tutukov, A. V. 1987, in this volume.

Jacoby, G. H., and Chu, Y.-H. 1987, in this volume.

Jacoby, G. H., and Ford, H. C. 1983, AP. J., 266, 298.

Jacoby, G. H., Quigley, R. J., and Africano, J. L. 1987, Pub. A. S. P., $\underline{99}, 672$.

Jewitt, D. C., Danielson, G. E., and Kupferman, P. N. 1986, Ap. J., 
$\underline{302}, 727$.

Kaler, J. B. 1974, A. J., 79, 594.

Kwok, S. 1983, in IAU Symp. No. 103, "Planetary Nebulae", ed. D. R.

Flower, (Reidel: Dordrecht), p. 293.

Kwok, S. 1987, in "Late Stages of Stellar Evolution", eds. S. Kwok and

S. Pottasch, (Reide1: Dordrecht), p. 321.

Lutz, J., Lame, N. J., and Balick, B. 1987, in this volume.

Manchado, A., Pottasch, S. R., and Mampaso, A. 1987a, in this volume. . 1987b, in this volume.

Mathews, W. G. 1978, in IAU Symp. No. 76, "Planetary Nebulae", ed.

Y. Terzian (Reidel: Dordrecht), p. 251.

Middlemass, D., Clegg, R. E. S., and Walsh, J. R. 1987, in this volume.

Millikan, A. G. 1974, A. J., 79, 1259.

Minkowski, R., and Osterbrock, D. 1960, Ap. J., 131, 537.

Moreno, M. A., and López, J. A. 1987, Astr. Ap., 178, 319.

O'Dell, C. R., and Ball, M. E. 1985, Ap. J., 289, 526.

Perek, L., and Kohoutek, L. 1987, Catalogue of Galactic Planetary Nebulae (Prague: Czechoslovaak Academy of Science)

Pismis, P., and Moreno, M. A. 1987, in this volume.

Reay, N. K., Atherton, P. D., and Taylor, K. 1983a, M. N. R. A. S., $\underline{203}, 1079$. . 1983b, M. N. R. A. S., 203, 1087.

Rosado, M. 1986, Rev. Mexicana. Astr. Ap., 13, 49.

Ruiz, M. T., Heathcote, S. R., and Weller, W. G. 1987, in this volume.

Sabbadin, F. 1984, Astr. Ap. Suppl., 58, 273.

Schmidt-Voigt, M., and Köppen, J. 1987a, Astr. Ap., 174, 211. . 1987b, Astr. Ap., 174, 223.

Walsh, J. R., and Meaburn, J. 1987, M. N. R. A. S., 224, 885.

Webster, B. L. 1978, M. N. R. A. S., 185, 45p.

Weller, W. G., and Heathcote, S. R. 1987a, in "Late Stages of Stellar Evolution", eds. S. Kwok and S. R. Pottasch, (Reidel: Dordrecht), p. 409. . 1987b, in this volume.

We1ty, D. E. 1983, Pub. A. S. P., 95, 217. 


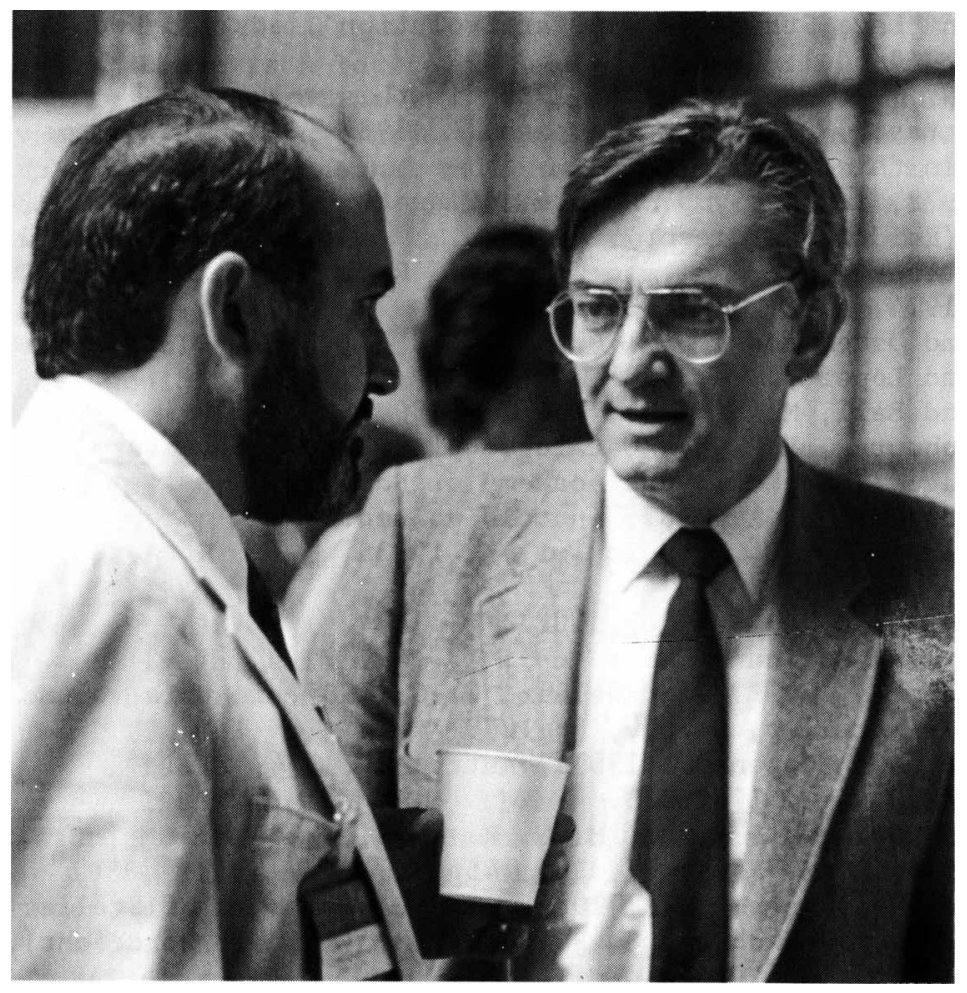

Manuel Peimbert and Luis F. Rodriguez. 